\title{
Thermal effect of surface tension on the inward solidification of spheres
}

\author{
T. Wu *, H.-C. Liaw, Y.-Z. Chen \\ Department of Mechanical Engineering, National Taiwan University, Taipei 106, Taiwan \\ Received 13 April 2001; received in revised form 21 September 2001
}

\begin{abstract}
Effect of surface tension (Gibbs-Thomson effect) on the inward solidification of a liquid in a spherical container is investigated analytically by solving the unsteady heat equation via a small-time series expansion technique. A nonlinear Shanks transformation is adopted to improve the convergence property of the series solution at large time. The results show that at fixed Stefan number, the effect of surface tension is to increase the growth rate of the freezing front. A local minimum in the freezing rate is found to develop for all surface tension parameter values considered in this study. Also, analytic expressions for the relations between the growth rate of the freezing front, Stefan number and surface tension parameter are derived under the asymptotic condition of small Stefan number. (c) 2002 Elsevier Science Ltd. All rights reserved.
\end{abstract}

\section{Introduction}

Solidification is a common process in many practical engineering applications such as refrigeration, ice formation, casting of alloys, and crystallization of pure substances. Generally speaking, such a process is featured by the existence of a moving interface between different phases at which the thermal energy in the form of latent heat is liberated. Owing to the unknown location of this solid/liquid interface and the nonlinear form of the thermal energy balance condition at the interface, analytical solutions are difficult to obtain except for few simple configurations. One of the few earliest exact solutions is that by Neumann and separately, by Stefan (see [1]) who studied the cooling of fluid from one end of a one-dimensional semi-infinite region. They obtained a closed solution for the temperature distribution in a selfsimilar form and deduced that the location of the interface advances with the square root of time. The specific solution has since been the starting point for the subsequent numerous approximate solutions to other types of boundary conditions and geometries. Hence-

\footnotetext{
${ }^{*}$ Corresponding author. Tel.: +886-2-236-302-31x2413; fax: +886-2-236-317-55.

E-mail address: tywu@ccms.ntu.edu.tw (T. Wu).
}

forth, the problems involving phase changes and moving interfaces were traditionally categorized as 'Stefan problems'.

References relevant to the one-dimensional Stefan problem were well documented in the book by Hill [2]. Specifically, various techniques and approximations have been attempted to study the problem of an inward solidification with spherical symmetry. These include the power-series approximation method of Kreith and Romie [3]; the closed-form solution by Langford [4] under the assumption of constant solidification rate; the approximate integral method of Poots [5]; the numerical solution by Tao [6], Li [7], and Caldwell and Chan [8]; the perturbation method by Huang and Shih [9]; the small parameter expansion solution by Pedroso and Domoto [10]; the strained coordinates method by Pedroso and Domoto [11]; the matched asymptotic expansions solution by Riley et al. [12]; the asymptotic solution (near the final stage of complete solidification) of Stewartson and Waechter [13]; the small-time expansion solutions by Davis and Hill [14], and Hill and Kucera [15].

However, all the above results did not take into account the thermal effect of the surface tension at the interface. Surface tension affects the equilibrium temperature (freezing or fusion temperature) at the interface of the two phases via the so-called Gibbs-Thomson law. If 


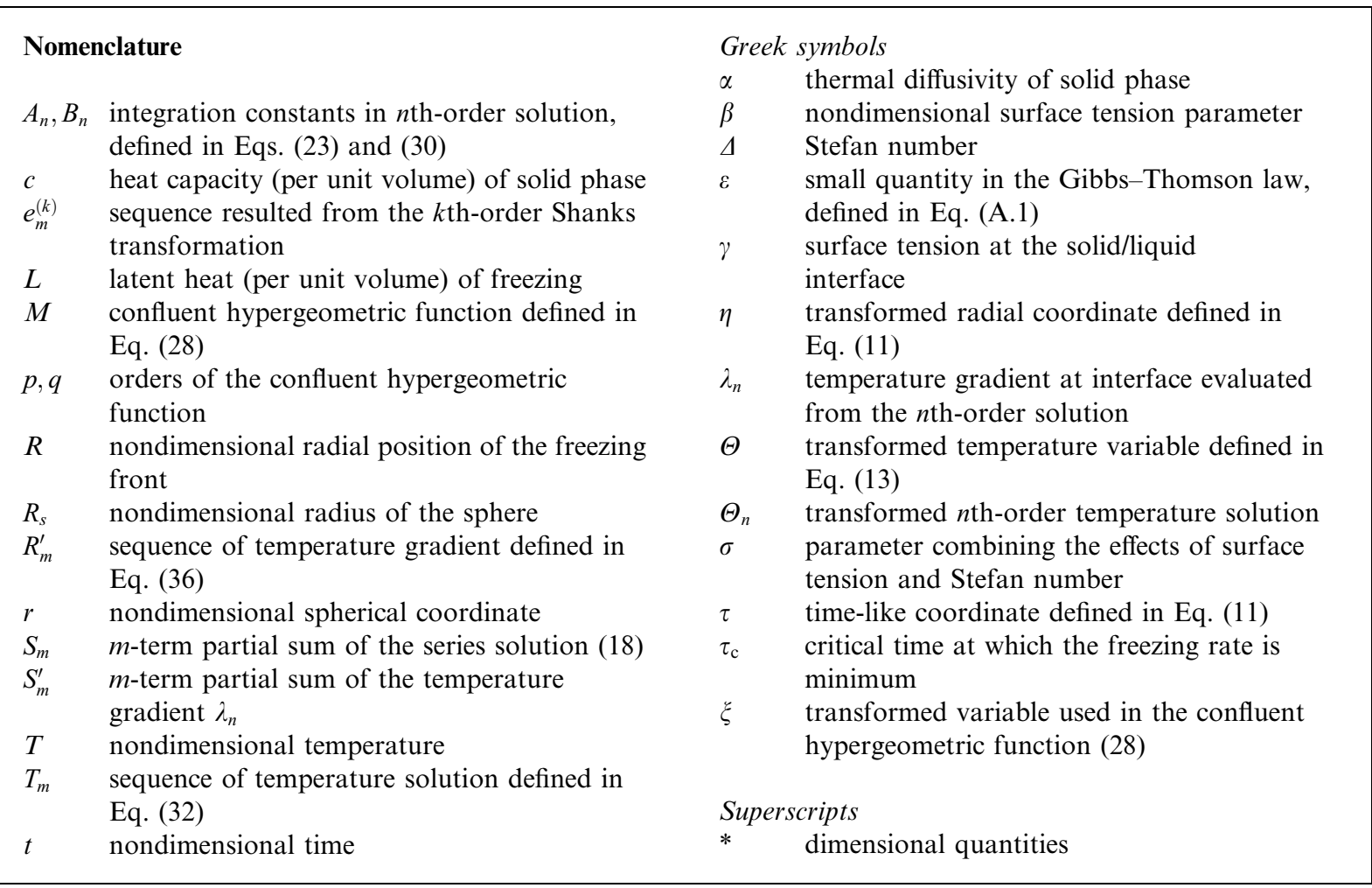

we let $\gamma$ denote the surface tension coefficient, $L$ the latent heat per unit volume of the solid phase, $R^{*}$ the radius of the spherical interface, and $T_{\mathrm{f}}^{*}$ the freezing/fusion temperature when the interface is flat; then the equilibrium temperature on the spherical freezing front is given by the following Gibbs-Thomson condition ([16]):

$T^{*}=T_{\mathrm{f}}^{*}\left(1+\frac{2 \gamma}{L R^{*}}\right)$.

That is, the equilibrium temperature on a curved interface (with center of radius lies in the liquid phase) is higher than that on a planar surface, and is increasing with decreasing radius of curvature of the interface. It should be noted that in deriving Eq. (1), a small value in $\left(T^{*}-T_{\mathrm{f}}^{*}\right) / T_{\mathrm{f}}^{*}$ has been assumed. Thus expression (1) is not valid when the second term $2 \gamma / L R^{*}$ in the parenthesis is of $\mathrm{O}(1)$, i.e., not suitable for freezing front with very small radius of curvature. In the present study, we shall investigate the inward freezing of liquid in a spherical domain (such as liquid in a spherical container, or a liquid droplet) under the modified equilibrium condition (1) as imposed by the surface tension at the interface.

As in all the previous works mentioned above, formulation of the present study is based on the classical one-phase model ([2]) that has been traditionally applied to most of the Stefan problems. In this one-sided ap- proach, the latent heat liberated from the freezing front is assumed to be carried away by the solidified phase only. This simplification leads to the familiar unsteady heat-diffusion equation for the solid phase with moving interfacial boundary. The equation describing the progression of the interface is derived from equating the amount of heat released per increase of the freezing layer to that diffused into the solidified field (Stefan condition). Since the Gibbs-Thomson condition is not valid when the freezing front comes near to the point of complete solidification, the set of equations is solved by means of a small-time series expansion technique. A nonlinear Shanks transformation ([17]) is applied to the series in order to improve the convergence of the solution at large time. The effect of surface tension on the temperature distribution in the solid field is discussed. Also, the asymptotic behavior of the freezing rate in terms of relevant physical parameters is determined to the leading-order approximation of small cooling parameter (Stefan number) value.

\section{Formulation of the problem}

The governing equation is that of the one-dimensional unsteady heat conduction equation written in the spherical coordinate $r^{*}$ 
$\frac{\partial T^{*}}{\partial t^{*}}=\alpha\left(\frac{2}{r^{*}} \frac{\partial T^{*}}{\partial r^{*}}+\frac{\partial^{2} T^{*}}{\partial r^{*^{2}}}\right), \quad R^{*} \leqslant r^{*} \leqslant R_{\mathrm{s}}^{*}$,

where $T^{*}$ represents the temperature, $\alpha$ is the thermal diffusion coefficient of the solid, $R_{s}^{*}$ denotes the radius of the sphere, and $R^{*}\left(t^{*}\right)$ the instantaneous location of the solid/liquid interface (see Fig. 1). Suppose that the temperature at the outer surface of the sphere is suddenly dropped to $T_{\mathrm{s}}^{*}$ below the freezing temperature at $t^{*}=0^{+}$and kept constant afterwards, then the proper boundary condition is

$T^{*}=T_{\mathrm{s}}^{*} \quad$ at $\quad r^{*}=R_{\mathrm{s}}^{*}$.

The equilibrium temperature at the interface is that due to the Gibbs-Thomson condition:

$T^{*}=T_{\mathrm{f}}^{*}\left(1+\frac{\gamma}{L} \frac{2}{R^{*}\left(t^{*}\right)}\right) \quad$ at $\quad r^{*}=R^{*}\left(t^{*}\right)$.

Balancing the heat releasing rate and the heat conduction rate at the freezing front gives the equation for the motion of the interface:

$L \frac{\mathrm{d} R^{*}}{\mathrm{~d} t^{*}}=\left.\alpha c \frac{\partial T^{*}}{\partial r^{*}}\right|_{R^{*}}$,

where $c$ denotes the heat capacity (per unit volume) of the solid phase.

Defining relevant dimensionless variables as

$T=\frac{T^{*}-T_{\mathrm{f}}^{*}}{T_{\mathrm{s}}^{*}-T_{\mathrm{f}}^{*}}, \quad r=\frac{r^{*}}{R_{\mathrm{s}}^{*}}, \quad R=\frac{R^{*}}{R_{\mathrm{s}}^{*}}, \quad t=\frac{\alpha t^{*}}{R_{\mathrm{s}}^{*^{2}}}$,

the nondimensional form of the Eq. (2) becomes

$\frac{\partial T}{\partial t}=\frac{2}{r} \frac{\partial T}{\partial r}+\frac{\partial^{2} T}{\partial r^{2}}, \quad R \leqslant r \leqslant 1$.

The associated boundary conditions are

$T=1 \quad$ at $\quad r=1$,

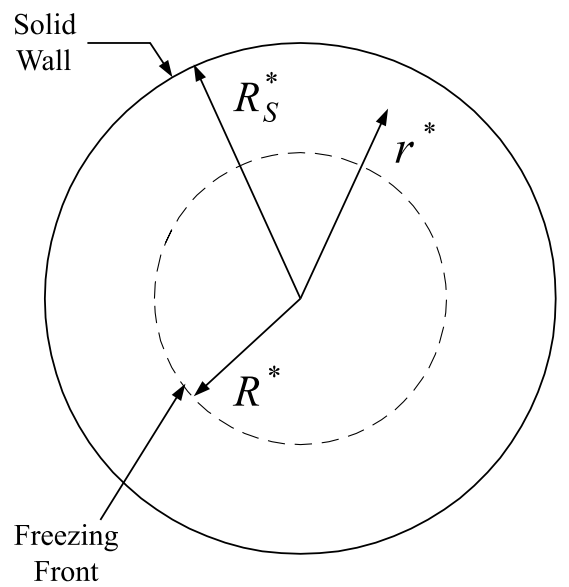

Fig. 1. Definition of the physical domain of the problem.
$T=-\frac{\beta}{\Delta} \cdot \frac{1}{R(t)}=-\frac{\sigma}{R(t)} \quad$ at $\quad r=R(t)$,

and the nondimensional growth rate of the freezing front is given by

$\frac{\mathrm{d} R}{\mathrm{~d} t}=-\left.\Delta \frac{\partial T}{\partial r}\right|_{R}$

In the above expressions, the dimensionless parameter $\Delta=c\left(T_{\mathrm{f}}^{*}-T_{\mathrm{s}}^{*}\right) / L$ is called the Stefan number, which measures the degree of cooling. The parameter $\beta=2 c \gamma T_{\mathrm{f}}^{*} /\left(R_{\mathrm{s}}^{*} L^{2}\right)$ represents the surface tension effect (Gibbs-Thomson effect), and $\sigma=\beta / \Delta$ is merely a convenient dimensionless parameter, which can also be taken as a measure of the surface tension when the Stefan number $\Delta$ is held fixed. Surface tension force is significant only when the radius of curvature is small. Water has a small surface tension coefficient $(\gamma=0.077$ $\mathrm{N} / \mathrm{m}$ ). Considering a liquid droplet of size $10^{-3} \mathrm{~m}$, the parameter $\sigma$ for water-ice system is then about $3 \times 10^{-4}$. Melted metals have large surface tension (e.g., $\gamma=1.9 \mathrm{~N} / \mathrm{m}$ for iron, $2.0 \mathrm{~N} / \mathrm{m}$ for nickel), typical values of $\sigma$ for common metals melts are around $10^{-2}$. In view of Eq. (9), surface tension has therefore a nonnegligible effect in solidification, particularly, when the radius of the freezing front $R(t)$ is small.

\section{Solution to the problem}

The problem involves a moving boundary (interface) whose position is part of the solution and hence is not known a priori. A boundary-fixed transformation [2]

$\eta=\frac{1-r}{1-R(t)}=\frac{1-r}{\tau(t)}$,

is adopted first to map the unknown position of the freezing front to a fixed value in $\eta, \eta=1$. In Eq. (11), $\tau=1-R$ is another variable describing the instantaneous position of the freezing front, which can also be treated as a time-like coordinate $(0 \leqslant \tau \leqslant 1)$. By using the chain rule, the original Eq. (7) can be rewritten in terms of the new coordinates $(\eta, \tau)$

$$
-\frac{\mathrm{d} R}{\mathrm{~d} t} \frac{\partial T}{\partial \tau}+\frac{\eta}{\tau} \frac{\mathrm{d} R}{\mathrm{~d} t} \frac{\partial T}{\partial \eta}=\frac{-2}{(1-\eta \tau) \tau} \frac{\partial T}{\partial \eta}+\frac{1}{\tau^{2}} \frac{\partial^{2} T}{\partial \eta^{2}} .
$$

A further transformation of temperature $T$ will put the equation in a form amenable to the series-expansion technique,

$T=\frac{\Theta}{r}=\frac{\Theta}{1-\eta \tau}$.

In terms of the new temperature variable $\Theta$, Eq. (12) becomes 
$-\tau^{2} \frac{\mathrm{d} R}{\mathrm{~d} t} \frac{\partial \Theta}{\partial \tau}+\eta \tau \frac{\mathrm{d} R}{\mathrm{~d} t} \frac{\partial \Theta}{\partial \eta}=\frac{\partial^{2} \Theta}{\partial \eta^{2}}$.

The corresponding boundary and interface conditions are

$\Theta=0$ at outer surface $\eta=0$,

$\Theta=-\sigma$ at interface $\eta=1$,

and the growth rate of the interface is governed by

$\frac{\mathrm{d} R}{\mathrm{~d} t}=\frac{\Delta}{\tau}\left(\left.\frac{1}{1-\tau} \frac{\partial \Theta}{\partial \eta}\right|_{\eta=1}+\left.\frac{\tau}{(1-\tau)^{2}} \Theta\right|_{\eta=1}\right)$.

The forms of Eqs. (14) and (17) suggest that we can expand the solution into a power series of the time-like variable $\tau$, i.e.,

$\Theta(\eta, \tau)=\sum_{n=0} \tau^{n} \Theta_{n}(\eta)$

Substituting (18) into Eqs. (14) and (17) and collecting the like-power terms for each different order of $\tau$, we obtain the following system of equations:

$$
\begin{aligned}
& \Theta_{n}^{\prime \prime}+\Delta \lambda_{0}\left[-\eta \Theta_{n}^{\prime}+n \Theta_{n}\right] \\
&=\Theta_{n-1}^{\prime \prime}+\Delta\left\{\sum_{k=0}^{n-1} \eta\left(\lambda_{k+1}-\sigma\right) \Theta_{n-k-1}^{\prime}\right. \\
&\left.-\sum_{k=0}^{n-2}(n-k-1)\left(\lambda_{k+1}-\sigma\right) \Theta_{n-k-1}\right\} \\
& n \in I, \quad n \geqslant 0,
\end{aligned}
$$

where $\sum_{k=0}^{m}(\cdot)=0$ is defined whenever $m<k$, and $\lambda_{n}=\left.\left(\mathrm{d} \Theta_{n} / \mathrm{d} \eta\right)\right|_{\eta=1}$ denotes the temperature gradient evaluated at the interface at each different order $n$. In Eq. (19), the prime symbols are shorthand notations for differentiations with respect to the coordinate $\eta$. The corresponding boundary conditions at each order $n$ are

$\Theta_{0}(0)=1, \quad \Theta_{n}(0)=0$ for $n \geqslant 1$,

$\Theta_{0}(1)=-\sigma, \quad \Theta_{n}(1)=0 \quad$ for $n \geqslant 1$.

The solution to the zeroth-order equation is easily obtained as

$\Theta_{0}=A_{0} \int_{0}^{\eta} \mathrm{e}^{\left(\Delta \lambda_{0} / 2\right) \eta^{2}} \mathrm{~d} \eta+B_{0}$,

where

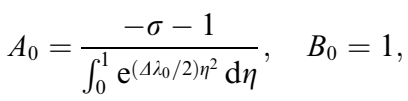

are the two integration constants determined from the boundary conditions (20) and (21). The temperature gradient at the interface is then obtained through differentiating Eq. (22): $\left.\lambda_{0} \equiv \frac{\mathrm{d} \Theta_{0}}{\mathrm{~d} \eta}\right|_{\eta=1}=\frac{-(1+\sigma)}{\int_{0}^{1} \mathrm{e}^{\left(\Delta \lambda_{0} / 2\right) \eta^{2}} \mathrm{~d} \eta} \mathrm{e}^{\left(\Delta \lambda_{0} / 2\right)}$.

Notice that the temperature gradient $\lambda_{0}$ appears at both sides of Eq. (24). An iterative procedure is then required to calculate the numerical value of $\lambda_{0}$.

The homogeneous parts of all subsequent higherorder equations $(n \geqslant 1)$ can be recast into a normal form via the coordinate transformation $\xi=\Delta \lambda_{0} \eta^{2} / 2$

$\xi \Theta_{n}^{\prime \prime}+\left(\frac{1}{2}-\xi\right) \Theta_{n}^{\prime}+\frac{n}{2} \Theta_{n}=0$.

Equation (25) falls into the class of the confluent hypergeometric equation ([18]), whose general form is

$\xi \Theta^{\prime \prime}+(q-\xi) \Theta^{\prime}-p \Theta=0$.

The two independent solutions to the above equation are

$M(p, q, \xi) \quad$ and $\quad \xi^{1-q} M(p-q+1,2-q, \xi)$

in which $M(p, q, \xi)$ is the confluent hypergeometric function of order $(p, q)$ defined as

$M(p, q, \xi)=\sum_{k=0}^{\infty} \frac{(p)_{k}}{(q)_{k}} \frac{\xi^{k}}{k !}$

with

$(p)_{k}=p \cdot(p+1) \cdot(p+2) \cdots(p+k-1)$ for $k \geqslant 1$,

$(q)_{k}=q \cdot(q+1) \cdot(q+2) \cdots(q+k-1)$ for $k \geqslant 1$,

$(p)_{0}=1, \quad(q)_{0}=1$.

Note that the power series in (28) terminates at finite terms for integer value of $p$. The solution to our present Eq. (25) corresponds to the case $(p, q)=(-n / 2,1 / 2)$. For certain special values of $(p, q)$, the hypergeometric function (28) can be related to the more familiar exponential and error functions [18]; e.g.

$M(p, p, \xi)=e^{\xi}$ for any number $p$,

$M\left(\frac{1}{2}, \frac{3}{2}, \xi\right)=\frac{1}{\sqrt{-\xi}} \int_{0}^{\sqrt{-\xi}} \mathrm{e}^{-z^{2}} \mathrm{~d} z=\frac{\sqrt{\pi}}{2 \sqrt{-\xi}} \operatorname{erf}(\sqrt{-\xi})$.

Thus by using proper recursive formulae ([18]) between different orders of the hypergeometric function $M(p, q, \xi)$, solution $\Theta_{n}$ to the homogeneous Eq. (25) can be rewritten into polynomial and error functions in $\eta$. Having obtained the solution to the homogeneous part of Eq. (19) for all $n$, the particular solution at each different order $n$ can be found by using the method of variation of parameters. The complete solution at each order $n$ is then the sum of the two.

Since the expression of the solution becomes rather lengthy and cumbersome very quickly with increasing 
order $n$, only the solution corresponding to the first order in $\tau$ (i.e. $\mathrm{O}(\tau)$ ) is given here:

$$
\begin{aligned}
\Theta_{1}= & A_{1} \eta-B_{1}\left[\mathrm{e}^{\left(\Delta \lambda_{0} / 2\right) \eta^{2}}-\Delta \lambda_{0} \eta \int_{0}^{\eta} \mathrm{e}^{\left(\Delta \lambda_{0} / 2\right) \eta^{2}} \mathrm{~d} \eta\right] \\
& +\frac{A_{0}}{3 \lambda_{0}}\left(\lambda_{0}+\lambda_{1}-\sigma\right) \eta \mathrm{e}^{\left(\Delta \lambda_{0} / 2\right) \eta^{2}}
\end{aligned}
$$

with integration constants

$A_{1}=\frac{-A_{0}}{3 \lambda_{0}}\left(\lambda_{0}+\lambda_{1}-\sigma\right) \mathrm{e}^{\left(\Delta \lambda_{0} / 2\right)}, \quad B_{1}=0$.

At this order, the temperature gradient $\lambda_{1}$ at the interface is given by

$\lambda_{1}=\left.\frac{\mathrm{d} \Theta_{1}}{\mathrm{~d} \eta}\right|_{\eta=1}=\frac{\left(A_{0} \Delta / 3\right)\left(\lambda_{0}-\sigma\right) \mathrm{e}^{\left(\Delta \lambda_{0} / 2\right)}}{1-\left(A_{0} \Delta / 3\right) \mathrm{e}^{\left(\Delta \lambda_{0} / 2\right)}}$.

In Eq. (31), $\lambda_{1}$ is an explicit function of $\lambda_{0}$ and $A_{0}$, so is the integration constant $A_{1}$. Thus having determined the values of $\lambda_{0}$ and $A_{0}$ from the lowest-order solution, all temperature gradients $\lambda_{n}$ and integration constants $A_{n}$ of the subsequent higher-order solutions can be obtained explicitly in terms of theses fundamental values.

In the present study, the solution has been determined up to $\mathrm{O}\left(\tau^{4}\right)$. We shall omit their expressions for brevity.

\section{Results and discussion}

\subsection{Temperature profiles}

We first define temperature solutions of increasingly higher orders by a sequence $T_{m}$

$T_{m}=\frac{S_{m}}{1-\eta \tau}$,

where $S_{m}=\sum_{n=0}^{m} \tau^{n} \Theta_{n}$ denotes the first $m$-term partial sum of the series solution (18). Successive $T_{m}$ indicate that terms of next order of $\tau$ are added to the solution series. In our present study, the value of $m$ is from 0 to 4 . Fixing the parameter values at $\Delta=0.1, \sigma=0.5$, Figs. 2 and 3 plot the sequences of temperature profiles $T_{m}$ at time $\tau=0.1$ and 0.75 (which correspond to $10 \%$ and $75 \%$ of solidification of the sphere), respectively. It can be seen that the series converges very fast at small time. The convergence rate of the series solution deteriorates gradually for large $\tau$, as shown in the insert of Fig. 3, which is a local magnification of the temperature distribution. Owing to the slow convergence, a large number of terms are needed in the expansion (18) to acquire a solution with satisfactory accuracy.

In order to improve the convergence rate of the series solution so that it can be applied to a much broader range of $\tau$, a nonlinear transformation proposed by

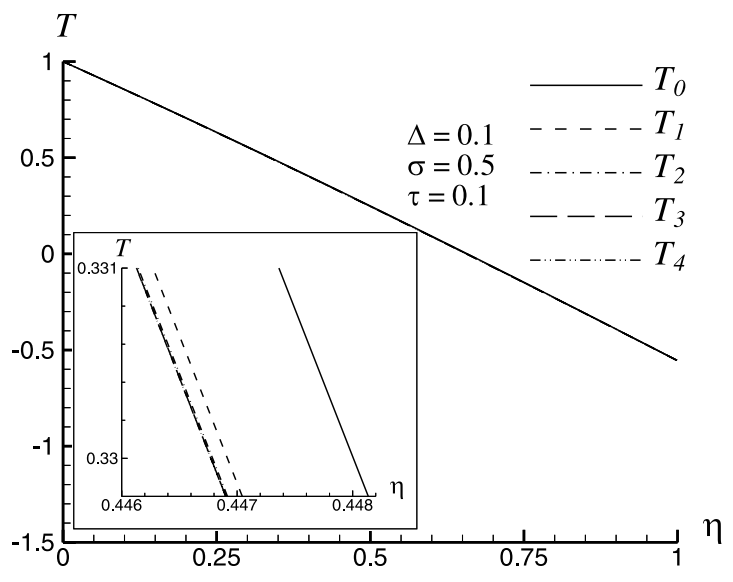

Fig. 2. Sequence of instantaneous temperature profiles $T_{m}$ at $\tau=0.1$.

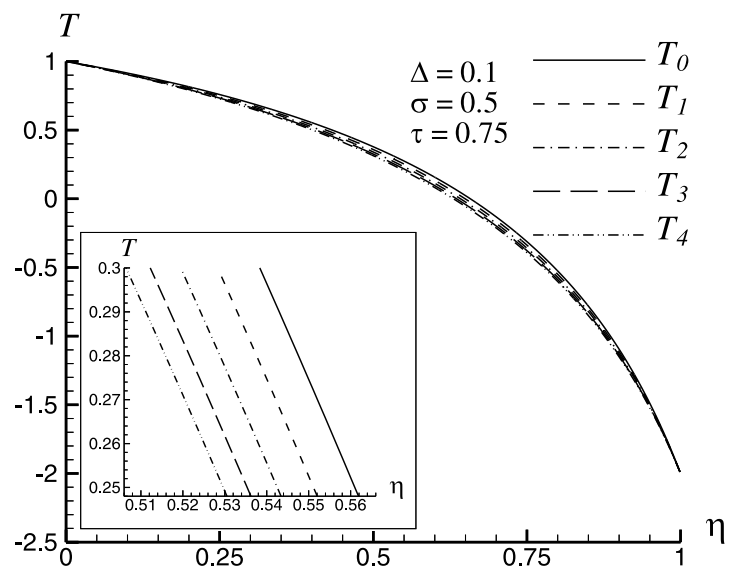

Fig. 3. Sequence of instantaneous temperature profiles $T_{m}$ at $\tau=0.75$.

Shanks [17] is adopted. Applying the first-order Shanks transformation results in a new sequence $e_{m}^{(1)}$ which is related to the original $T_{m}$ by the following formula:

$e_{m}^{(1)}(T)=T_{m+1}-\frac{\left(T_{m+2}-T_{m+1}\right)\left(T_{m+1}-T_{m}\right)}{\left(T_{m+2}-T_{m+1}\right)-\left(T_{m+1}-T_{m}\right)}$,
$m \geqslant 0$.

The same transformation rule can be again applied on the newly formed sequence $e_{m}^{(1)}$ to yield a second new sequence $e_{m}^{(2)}$ :

$e_{m}^{(2)}(T)=e_{m+1}^{(1)}-\frac{\left(e_{m+2}^{(1)}-e_{m+1}^{(1)}\right)\left(e_{m+1}^{(1)}-e_{m}^{(1)}\right)}{\left(e_{m+2}^{(1)}-e_{m+1}^{(1)}\right)-\left(e_{m+1}^{(1)}-e_{m}^{(1)}\right)}$,

Expression (34) is termed the second-order Shanks transformation. The process can be continued to 
produce still higher-order Shanks transformation sequences. In the present case, the original temperature sequence $T_{m}$ is up to $m=4$ only; which allows for at most the employment of the second-order Shanks transformation. Connections between the original sequence $T_{m}$ and the sequences generated by applying the first- and second-order Shanks transformations, $e_{m}^{(1)}(T)$ and $e_{m}^{(2)}(T)$ are most instructively represented by the following triangular arrays:

$$
\begin{array}{lll}
T_{0} & & \\
T_{1} & & \\
T_{2} & e_{0}^{(1)}(T) & \\
T_{3} & e_{1}^{(1)}(T) & \\
T_{4} & e_{2}^{(1)}(T) & e_{0}^{(2)}(T) .
\end{array}
$$

According to Shanks, the sequence formed by elements along the diagonal line has a much better convergence property than the original one. In most cases, we may even obtain an induced convergent sequence from an originally divergent one through the Shanks transformation. Notion of employing the rational fraction (33) to approximate a geometric series and the convergence property of the transformed diagonal sequence were discussed in detail by Shanks [17]. Figure 4 shows at $\tau=0.75$ the sequence of temperature profiles constructed from the diagonal elements $T_{0}, e_{0}^{(1)}(T)$, and $e_{0}^{(2)}(T)$ of the above triangular arrays. Clearly, the convergence rate is improved in this new sequence. Figure 5 compares at $\tau=0.75$ the original sequence of temperature profile $T_{m}$ with that obtained from applying the second-order Shanks transformation, $e_{0}^{(2)}(T)$. If we consider $e_{0}^{(2)}(T)$ a more accurate result, apparently, the original solution (18) ought to be expanded to a much higher order of $\tau$.

Instantaneous temperature profiles for different surface tension values $\sigma$ and Stefan numbers $\Delta$ are plotted in Figs. 6 and 7. All temperature profiles shown in these

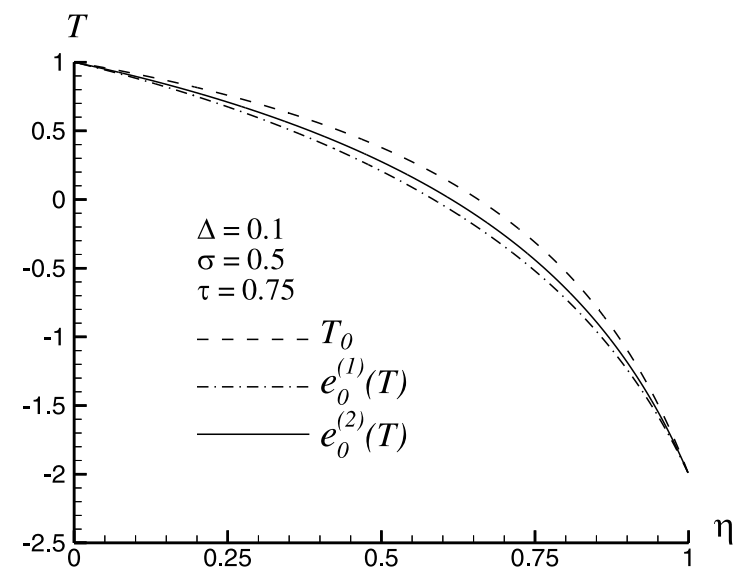

Fig. 4. Sequence of temperature profiles constructed from applying the first- and second-order Shanks transformations.

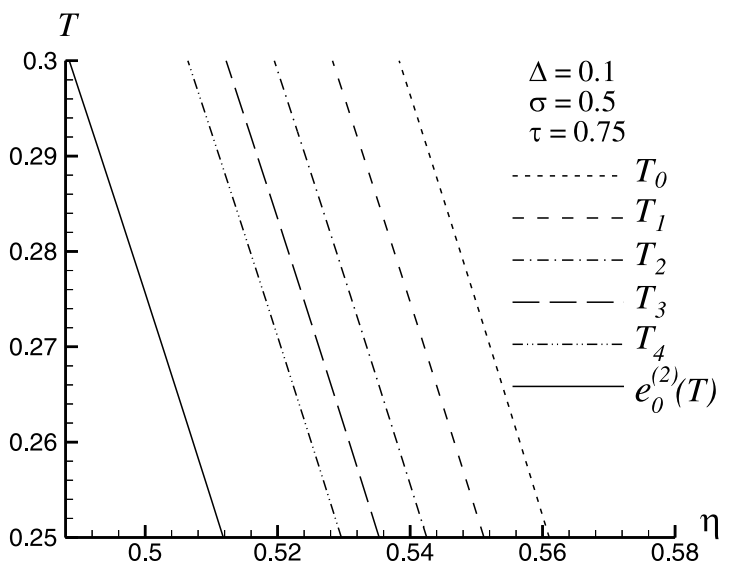

Fig. 5. Comparison of the sequence of temperature profiles $T_{m}$ with the more accurate result obtained from applying the second-order Shanks transformation.

plots are that from the second-order Shanks transformation, $e_{0}^{(2)}(T)$. In reading these plots, it is reminded that in the definition of the dimensionless temperature $T$ (Eq. (6)), $T_{\mathrm{s}}^{*}-T_{\mathrm{f}}^{*}$ is a negative quantity (since it is a freezing process). Thus the larger the value of the dimensionless temperature $T$ is, the lower is the physical temperature $T^{*}$. Figure 6 indicates that the physical temperature profile $T^{*}$ has a larger value everywhere in the solidified layer for larger surface tension value. This is not surprising on viewing the interface condition (9), which states that the action of the surface tension (under fixed Stefan number) is to increase the equilibrium temperature at the interface. The same result is found in Fig. 7 where the surface tension $\beta$ is held fixed; the dimensional temperature profile $T^{*}$ has a higher value for smaller Stefan number $\Delta$.

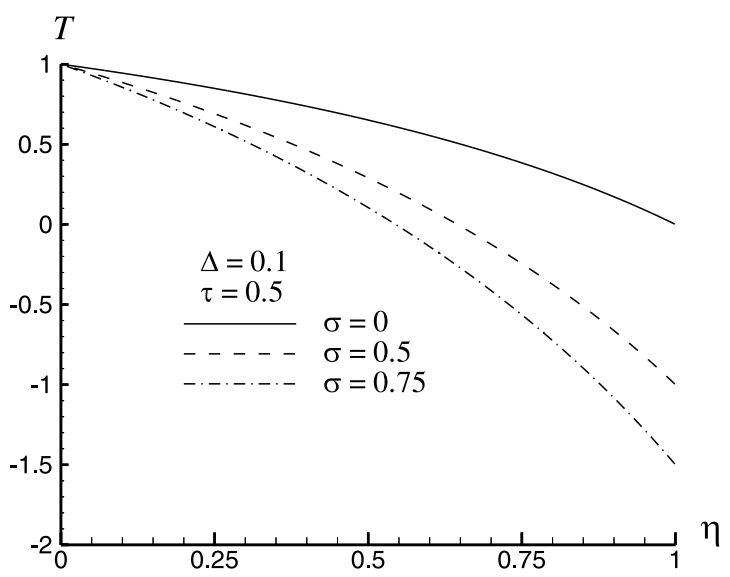

Fig. 6. Instantaneous temperature profiles at $\tau=0.5$ under different surface tension values. 


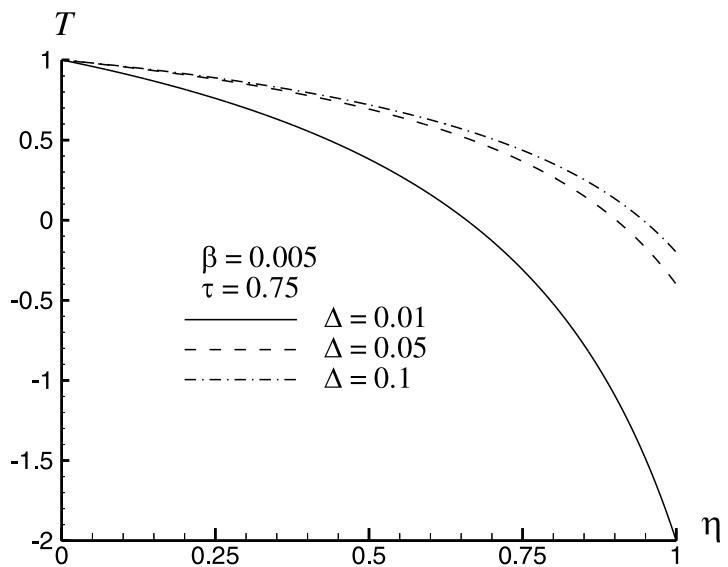

Fig. 7. Instantaneous temperature profiles at $\tau=0.75$ under different Stefan number values.

\subsection{Freezing rate}

Another quantity that is of interest is the freezing rate, i.e., the growth rate of the interface,

$$
\begin{aligned}
\frac{\mathrm{d} R}{\mathrm{~d} t}=\frac{\Delta}{\tau}( & \frac{\lambda_{0}+\tau \lambda_{1}+\tau^{2} \lambda_{2}+\tau^{3} \lambda_{3}+\tau^{4} \lambda_{4}+\cdots}{1-\tau} \\
& \left.-\frac{\tau \sigma}{(1-\tau)^{2}}\right)
\end{aligned}
$$

As before, we may define a sequence representing different orders of the freezing rate solutions by successively adding the next higher order terms into the expression

$R_{m}^{\prime}=\frac{\Delta}{\tau}\left(\frac{S_{m}^{\prime}}{1-\tau}-\frac{\tau \sigma}{(1-\tau)^{2}}\right)$,

where $S_{m}^{\prime}=\sum_{n=0}^{m} \tau^{n} \lambda_{n}$ denotes the first $m$-term partial sum of the temperature gradient $\lambda_{n}$ obtained from the series solution (18). Once again, we may apply the firstand second-order Shanks transformations to achieve better-converged results from the above sequence $R_{m}^{\prime}$. In analogy to Eqs. (33) and (34), we have

$$
\begin{gathered}
e_{m}^{(1)}\left(R^{\prime}\right)=R_{m+1}^{\prime}-\frac{\left(R_{m+2}^{\prime}-R_{m+1}^{\prime}\right)\left(R_{m+1}^{\prime}-R_{m}^{\prime}\right)}{\left(R_{m+2}^{\prime}-R_{m+1}^{\prime}\right)-\left(R_{m+1}^{\prime}-R_{m}^{\prime}\right)}, \\
m \geqslant 0, \\
e_{m}^{(2)}\left(R^{\prime}\right)=e_{m+1}^{(1)}-\frac{\left(e_{m+2}^{(1)}-e_{m+1}^{(1)}\right)\left(e_{m+1}^{(1)}-e_{m}^{(1)}\right)}{\left(e_{m+2}^{(1)}-e_{m+1}^{(1)}\right)-\left(e_{m+1}^{(1)}-e_{m}^{(1)}\right)}, \\
m \geqslant 0 .
\end{gathered}
$$

Figure 8 shows the evolution history of the freezing rate $R_{m}^{\prime}$ with parameters values $\Delta=0.1$ and $\sigma=0.5$,

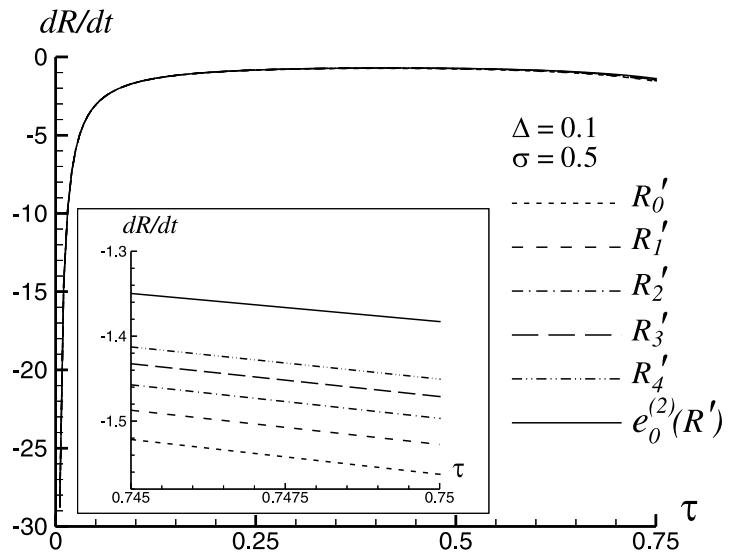

Fig. 8. Evolution history of the freezing rate solution sequence $R_{m}^{\prime}$.

Ewhile plotted along is the improved result obtained from the second-order Shanks transformation $e_{0}^{(2)}\left(R^{\prime}\right)$ for comparison. Convergence of the sequence $R_{m}^{\prime}$ is quite good except at large $\tau$. Slow convergence of the original sequence $R_{m}^{\prime}$ at large time is shown in the insert of Fig. 8. Figure 9 gives the history of the freezing rate (after Shanks transformation) under various surface tension values. As indicated by the figure, raising the surface tension at the interface expedites the freezing rate $|\mathrm{d} R / \mathrm{d} t|$. In all cases demonstrated here, the freezing rate drops sharply at the early stage of solidification, and then becomes leveling off progressively. It is found that at certain critical time the freezing rate reaches a minimum, after that, it rises again. Basically, the freezing rate in this problem is governed by two mechanisms. Thickening of the solidified layer reduces the temperature gradient across the layer and hence reducing the growth rate of the interface; while the ever rising of the

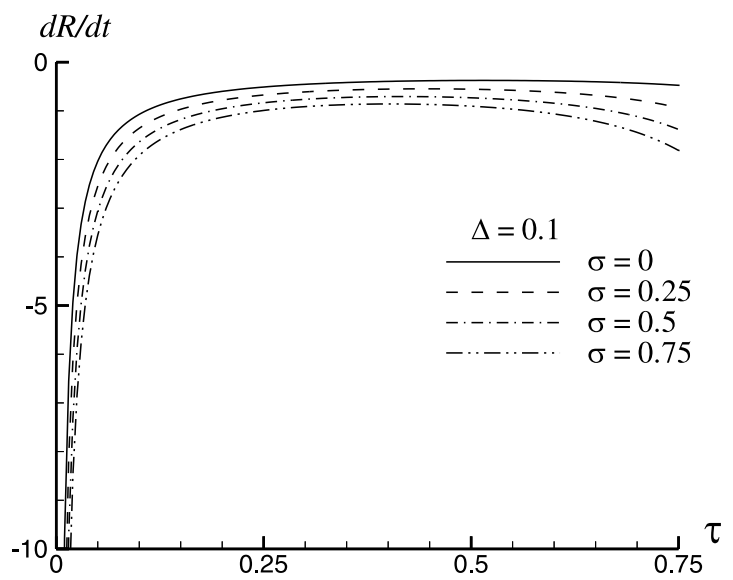

Fig. 9. Evolution history of the freezing rate under various surface tension conditions. 
equilibrium temperature at the interface as the freezing front progresses towards the center of the sphere (as given by the Gibbs-Thomson condition (9)) causes an increase in the temperature gradient at the interface and hence increasing the freezing rate. The latter effect is more pronounced near the completion of freezing when the radius of the interface is close to zero. Competition of these two opposite effects results in the observed dropping and rising of the freezing rate at the initial and final phases of solidification, respectively.

\subsection{Asymptotic results of freezing rate for $\Delta \ll 1$}

Figure 8 suggests that the temperature-gradient series $S_{m}^{\prime}$ in (36) can be truncated at $\mathrm{O}\left(\tau^{o}\right)$ term, the resulting expression for the freezing rate

$\frac{\mathrm{d} R}{\mathrm{~d} t} \approx R_{0}^{\prime}=\frac{\Delta}{\tau}\left[\frac{\lambda_{0}}{1-\tau}-\frac{\tau \sigma}{(1-\tau)^{2}}\right]$

is still a good approximation, at least up to a moderate value of time $\tau$. It is recalled that the $\lambda_{0}$ in (39) is an implicit function of $\Delta$ through Eq. (24). In most freezing and casting problems, the Stefan number $\Delta$ is usually small $\left(\Delta \sim \mathrm{O}\left(10^{-2}\right)\right)$, which enables the derivation of the leading-order expression of $\lambda_{0}$ for $\Delta \ll 1$. After expanding the exponential and error functions at the righthand-side of Eq. (24) for small $\Delta$, we have, correct to $\mathrm{O}(\Delta)$,

$\lambda_{0} \cong-(1+\sigma)+\frac{\Delta}{3}(1+\sigma)^{2}$.

On substituting (40) into Eq. (39), the analytical approximation of the growth rate is obtained as

$$
\begin{aligned}
\frac{\mathrm{d} R}{\mathrm{~d} t} \cong \frac{\Delta}{\tau}\{ & \frac{\Delta}{3(1-\tau)}(1+\sigma)^{2}-\frac{1}{(1-\tau)}(1+\sigma) \\
& \left.-\frac{\tau \sigma}{(1-\tau)^{2}}\right\} .
\end{aligned}
$$

Depending on the relative orders of magnitude of the parameters $\Delta$ and $\tau$, we may further distinguish the following three cases:

\section{(I) $\tau \ll \Delta \ll 1$ (approximation 1 )}

In this parameter range, Eq. (41) is dominated by the leading-order term

$\frac{\mathrm{d} R}{\mathrm{~d} t} \cong-\frac{\Delta}{\tau}(1+\sigma)$.

(II) $\tau \approx \Delta \ll 1$ (approximation 2 )

In this parameter range, Eq. (41) is simplified to (correct to $\mathrm{O}(\Delta)$ and $\mathrm{O}(\tau)$ )

$\frac{\mathrm{d} R}{\mathrm{~d} t} \cong-\frac{\Delta}{\tau}\left\{(1+\tau)(1+\sigma)+\tau \sigma-\frac{\Delta}{3}(1+\sigma)^{2}\right\}$.
(III) $\Delta \ll 1 \approx \tau$ (approximation 3 )

In this parameter range, the leading-order approximation of (41) is (correct to $\mathrm{O}(\Delta)$ )

$$
\begin{aligned}
\frac{\mathrm{d} R}{\mathrm{~d} t} & \cong-\frac{\Delta}{\tau}\left\{\frac{1}{1-\tau}(1+\sigma)+\frac{\tau \sigma}{(1-\tau)^{2}}\right\} \\
& =-\frac{\Delta}{\tau}\left\{\frac{1+\sigma-\tau}{(1-\tau)^{2}}\right\} .
\end{aligned}
$$

Eqations (42)-(44) provide in each different range of time an asymptotic expression of the freezing rate in terms of relevant physical parameters $\Delta, \sigma$ and $\tau$ under the condition of small Stefan number $\Delta \ll 1$. For instance, to leading-order approximation, the above asymptotic results predict a linear dependence of the freezing rate on the surface tension parameter $\sigma$ for all time ranges. The three expressions are plotted in Fig. 10 for $\Delta=0.01, \sigma=0.5$. In each plot, more accurate result from the second-order Shanks transformation of the original sequence $R_{m}^{\prime}$ is also provided for comparison. Clearly, both results are in excellent agreement, indicating that Eqs. (42)-(44) are indeed good approximations to the freezing rate in the respective parameters ranges. Particularly we note that in Fig. 10(c), the validity of the approximation 3 under the designated parameters values is shown to extend at least up to a time as large as $\tau=0.75$. In fact this can be easily justified by glancing at Eq. (31), we immediately come to the conclusion $\lambda_{1} \sim \mathrm{O}(\Delta)$ (and so for the subsequent $\lambda_{n}, n \geqslant 1$ ). Thus Eq. (44), which is derived from applying the oneterm approximation to the series in (35), is theoretically the correct leading-order expansion of the freezing rate for all fixed $\tau(\tau<1)$ under the asymptotic condition $\Delta \rightarrow 0$.

It has been mentioned previously that the growth rate of the interface exhibits a local minimum at certain critical time $\tau_{c}$ whose value depends on the surface tension parameter $\sigma$ and the Stefan number $\Delta$. Again, such a relationship can be derived from the approximate form of the freezing rate, Eq. (39). The condition for a local extreme to occur in the growth rate is

$$
\begin{aligned}
\frac{\mathrm{d}}{\mathrm{d} \tau}\left(\frac{\mathrm{d} R}{\mathrm{~d} t}\right) & =\frac{\Delta}{\tau^{2}(1-\tau)^{3}}\left[-\lambda_{0}(1-\tau)+2 \lambda_{0} \tau(1-\tau)-2 \sigma \tau^{2}\right] \\
& =0 .
\end{aligned}
$$

The above criterion leads to the finding of the roots to the following quadratic equation:

$$
\tau^{2}-\frac{3 \lambda_{0}}{2\left(\lambda_{0}+\sigma\right)} \tau+\frac{\lambda_{0}}{2\left(\lambda_{0}+\sigma\right)}=0
$$

Thus the critical time $\tau_{\mathrm{c}}$ at which the freezing rate is minimum is given by 


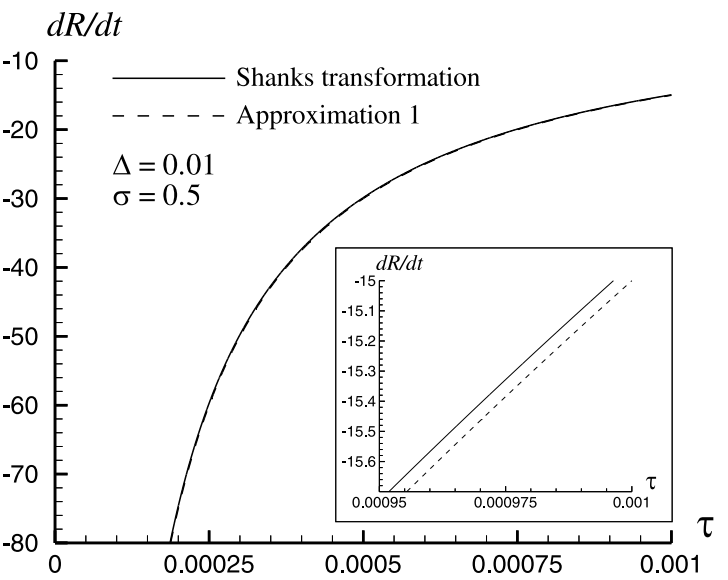

(a)
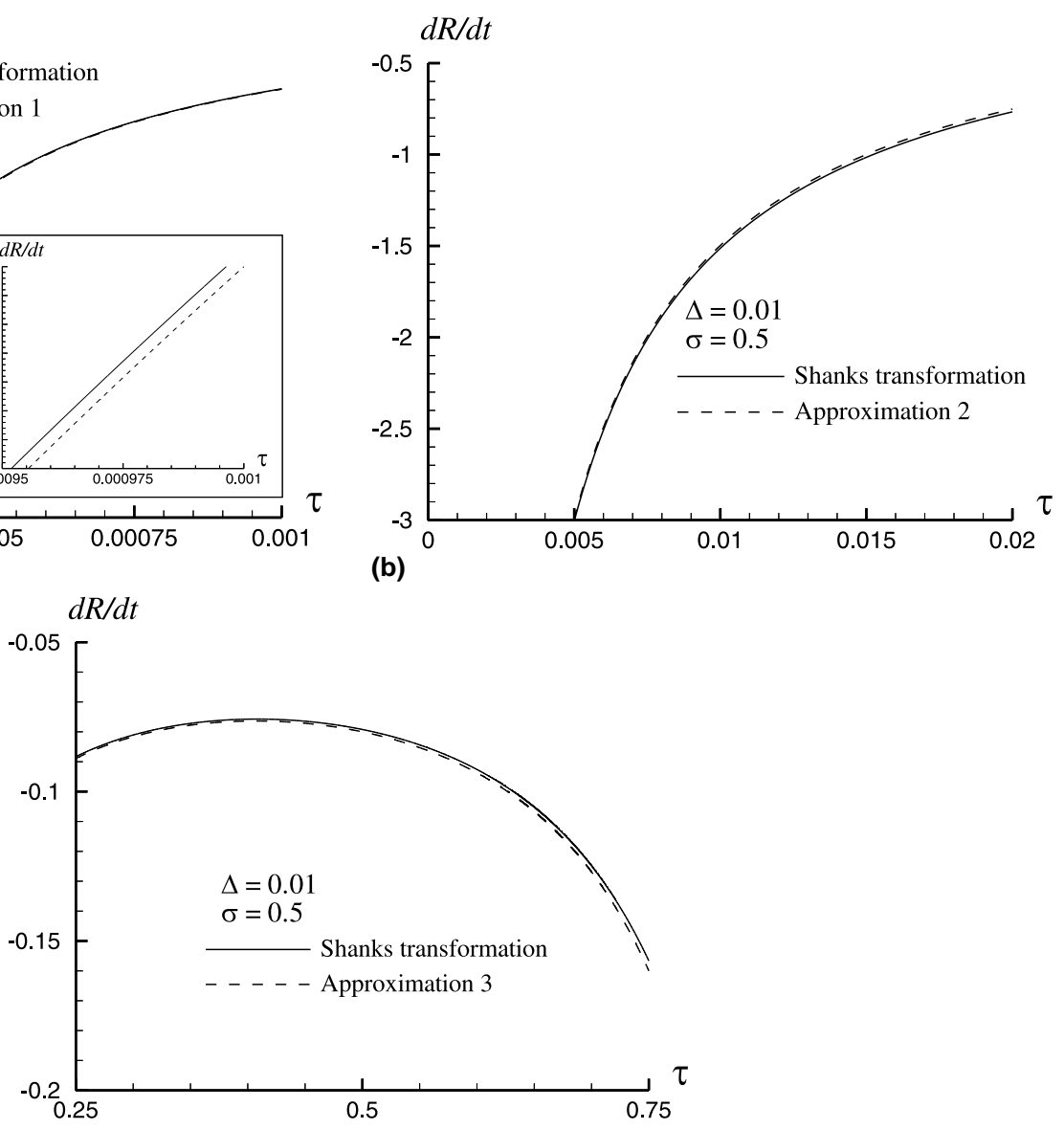

(c)

Fig. 10. Comparisons between the asymptotic results of the freezing rate and that obtained from the original series solution (with Shanks transformation) in the parameters ranges (a) $\tau \ll \Delta \ll 1$, (b) $\tau \approx \Delta \ll 1$, (c) $\Delta \ll 1 \approx \tau$.

$\tau_{\mathrm{c}}=\frac{3 \lambda_{0}}{4\left(\lambda_{0}+\sigma\right)} \pm \frac{\sqrt{\left(\lambda_{0}^{2}-8 \sigma \lambda_{0}\right) /\left(\lambda_{0}+\sigma\right)^{2}}}{4}$

in which the solution with ' + ' sign is to be discarded because it gives a time $\tau_{\mathrm{c}}$ greater than 1 (i.e. greater than the total time needed for a complete solidification). Substituting the asymptotic expression of $\lambda_{0}$ (Eq. (40)) into Eq. (45) and expanding it for $\Delta \ll 1$, we obtain the approximate expression for the critical time $\tau_{\mathrm{c}}$ (correct to $\mathrm{O}(\Delta))$

$$
\begin{aligned}
\tau_{\mathrm{c}}= & \frac{1}{4}[3(\sigma+1)-\sqrt{(\sigma+1)(9 \sigma+1)}]+\frac{\Delta}{4} \sigma(\sigma+1) \\
& \times\left[(\sigma+1)-\frac{(9 \sigma+5)}{3(9 \sigma+1)} \sqrt{(\sigma+1)(9 \sigma+1)}\right] .
\end{aligned}
$$

Equation (46) is graphed in Fig. 11 for various surface tension parameter values $\beta(\beta=\sigma \Delta)$ and Stefan number $\Delta$. It is observed that critical freezing rate occurs at an earlier time for larger surface tension value and

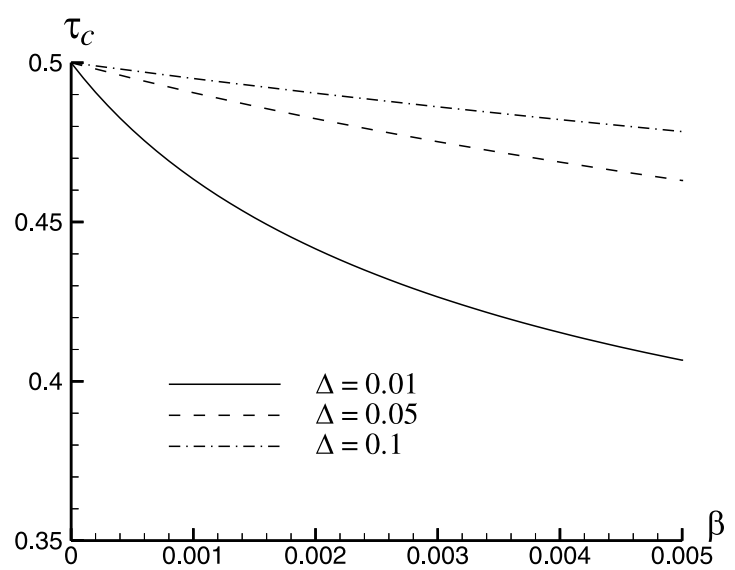

Fig. 11. Critical time $\tau_{\mathrm{c}}$ for the freezing rate as a function of surface tension parameter $\beta$ and Stefan number $\Delta$. 
smaller Stefan number - a situation corresponding to a higher equilibrium temperature (dimensional) at the solid/liquid interface (see the boundary condition (9)) and hence a higher temperature gradient at the freezing front. In all cases we have considered here, minimal freezing rate is found to develop in the first half of the solidification process, i.e., $\tau_{\mathrm{c}} \leqslant 0.5$. From Fig. 11 , it is also noticed that when $\beta=0$; the minimal freezing rate always occurs at $\tau_{\mathrm{c}}=0.5$, regardless the value of the Stefan number (as long as $\Delta \ll 1$ ). This is already implied in Eq. (39) as $\sigma=0$ is substituted into the equation; the resulting form of $\mathrm{d} R / \mathrm{d} t$ has an extreme at $\tau=0.5$, independent of $\Delta$.

\section{Concluding remarks}

A small-time series expansion technique has been applied in this work to study the thermal effect of the surface tension on an inward solidification process with spherical symmetry. A nonlinear Shanks transformation has been adopted to improve the convergence rate of the series solution so as to extend its applicability to a larger time range. In summary, the present result shows that surface tension increases the equilibrium temperature at the solid/liquid interface and hence speeds up the growth rate of the freezing front. The asymptotic expression of the freezing rate has been obtained for each different time range under the condition of small Stefan number. To leading-order approximation, the results predict a linear increase of the freezing rate with the surface tension parameter for all time ranges under consideration. Critical time at which the freezing rate develops a local minimum has also been determined analytically, which shows that the freezing rate reaches its minimum value more quickly for larger surface tension value at the interface.

The form of the Gibbs-Thomson law assumed in the present analysis is not applicable for solid/liquid interface with extremely small radius of curvature. Thus it is expected that the validity of the present result cannot be extended to the time when the freezing front comes very close to the center of the sphere. In practical applications, the upper limit of such time depends on the numerical values of various parameters. A simple estimation of a typical value of this limitation time is given in the appendix. In practice, the limitation is not that stringent as it may appear. Aside from this, the present study does provide critical information regarding to the variation of the freezing rate with relevant physical parameters as the thermal effect of the surface tension at the interface is taken into consideration. It is clear from the present analysis that surface tension has nonnegligible influence on the freezing rate. This is particularly true at the initial stage $(\tau \approx 0)$ and close to the final phase $(\tau \approx 1)$ of solidification where the freezing rate is shown to be augmented by a factor $\sigma / \tau$ and $\sigma /(1-\tau)^{2}$, respectively.

\section{Appendix A}

To estimate the typical value of $\tau$ beyond which the present analysis is not applicable, we start from the Gibbs-Thomson law, Eq. (1). Already, it has been stated in Section 1 that Eq. (1) holds only if $\varepsilon=2 \gamma / L R^{*} \ll 1$. Rewrite the condition using nondimensional parameters $\sigma$ and $R$ defined in the text, we obtain

$\varepsilon=\sigma\left(T_{\mathrm{f}}^{*}-T_{\mathrm{s}}^{*}\right) /\left(R T_{\mathrm{f}}^{*}\right) \ll 1$.

Let us take $\sigma=0.1, T_{\mathrm{f}}^{*}=273 \mathrm{~K}$; and assume a small degree of cooling, $\left(T_{\mathrm{f}}^{*}-T_{\mathrm{s}}^{*}\right)=5 \mathrm{~K}$ for example. Now, if we accept that 0.05 is practically a small enough number for $\varepsilon$, condition (A.1) then gives

$\tau=1-R \approx 0.96$,

which stands for a $96 \%$ of solidification of the sphere. Moreover, the smaller the surface tension parameter $\sigma$, the closer this value is to 1 . Thus in practice, limitation on the applicability of the Gibbs-Thomson law in the form of Eq. (1) is not that restricted as it may appear. In most cases involving small surface tension, small Stefan number or small degree of cooling, we can push the limit of usefulness of the present series solution to a point not too far from a complete solidification.

\section{References}

[1] H.S. Carslaw, J.C. Jaeger, Conduction of Heat in Solids, second ed., Clarendon Press, Oxford, 1959.

[2] J.M. Hill, One-Dimensional Stefan Problems: An Introduction, Longman Scientific and Technical, Essex, 1987.

[3] F. Kreith, F.E. Romie, A study of the thermal diffusion equation with boundary conditions corresponding to solidification or melting of materials initially at the fusion temperature, Proc. Phys. Soc. 68B (1955) 277-286.

[4] D. Langford, The freezing of spheres, Int. J. Heat Mass Transfer 9 (1966) 827-836.

[5] G. Poots, On the application of integral methods to the solution of problems involving the solidification of liquids initially at fusion temperature, Int. J. Heat Mass Transfer 5 (1962) 525-531.

[6] L.C. Tao, Generalized numerical solutions of freezing a saturated liquid in cylinders and spheres, AIChE J. 13 (1) (1967) 165-169.

[7] N.-Y. Li, Thermomechanical stresses and some asymptotic behavior in castings with spherical solidification, J. Thermal Stresses 18 (1995) 165-184.

[8] J. Caldwell, C.-C. Chan, Numerical solutions of the Stefan problem by the enthalpy method and the heat balance integral method, Numer. Heat Transfer Part B 33 (1998) 99-117. 
[9] C.L. Huang, Y.P. Shih, A perturbation method for spherical and cylindrical solidification, Chem. Eng. Sci. 30 (1975) 897-906.

[10] R.I. Pedroso, G.A. Domoto, Perturbation solutions for spherical solidification of saturated liquids, ASME J. Heat Transfer 95 (1973) 42-46.

[11] R.I. Pedroso, G.A. Domoto, Inward spherical solidification-solution by the method of strained coordinates, Int. J. Heat Mass Transfer 16 (1973) 1037-1043.

[12] D.S. Riley, F.T. Smith, G. Poots, The inward solidification of spheres and circular cylinders, Int. J. Heat Mass Transfer 17 (1974) 1507-1516.

[13] K. Stewartson, R.T. Waechter, On Stefan's problem for spheres, Proc. R. Soc. London Ser. A 348 (1976) 415-426.
[14] G.B. Davis, J.M. Hill, A moving-boundary problem for the sphere, IMA J. Appl. Math. 29 (1982) 99-111.

[15] J.M. Hill, A. Kucera, Freezing a saturated liquid inside a sphere, Int. J. Heat Mass Transfer 26 (1983) 1631-1637.

[16] D.T.J. Hurle (Ed.), Handbook of Crystal Growth Volume I: Fundamentals, North-Holland, Amsterdam, 1993, pp. 194-197.

[17] D. Shanks, Nonlinear transformations of divergent and slowly convergent sequences, J. Math. Phys. 34 (1955) $1-42$.

[18] M. Abramowitz, I.A. Stegun (Eds.), Handbook of Mathematical Functions, Dover, New York, 1965. 